\title{
Stationary Regimes of a Plasma Diode.
}

\author{
A. Sestero and M. Zannetti \\ Laboratorin Gas Ionizzati (Associazione EURATOM-CNEN) - Frascati \\ ( Nuovo Cimento, $51 \mathrm{~B}, 230(1967)$ )
}

The following corrections are brought to the reader's attention.

On page 231 , last line before eq. (5), the word "by" after the parenthesis is to be replaced by a colon sign (:).

On page 233 , 8 th and 9 th lines, the sentence between the words printed in italics and the words in parentheses is to be rephrased as follows: "... the reason being that the density of these particles in the plasma region is greater than that of the particles emitted from the other electrode ..." .

On page 234, 6th line, the indication "Fig. 1 " is to be replaced by "Fig. 3 ".

On page 234 , caption of Fig. 3 , the remark in parentheses "logarithmic density scale " does not belong there, but to Fig. 1. 